\title{
Spin and Wind Directions I: Identifying Entanglement in Nature and Cognition
}

\author{
Diederik Aerts ${ }^{1} \cdot$ Jonito Aerts Arguëlles $^{2}$ - Lester Beltran ${ }^{3} \cdot$ \\ Suzette Geriente ${ }^{4} \cdot$ Massimiliano Sassoli de Bianchi $^{1}$ • \\ Sandro Sozzo 5 - Tomas Veloz ${ }^{1,6}$
}

(C) The Author(s) 2017. This article is an open access publication

\begin{abstract}
We present a cognitive psychology experiment where participants were asked to select pairs of spatial directions that they considered to be the best example of Two different wind directions. Data are shown to violate the CHSH version of Bell's inequality with the same magnitude as in typical Bell-test experiments with entangled spins. Wind directions thus appear to be conceptual entities connected through meaning, in human cognition, in a similar way as spins appear to be entangled in experiments conducted in
\end{abstract}

Massimiliano Sassoli de Bianchi

msassoli@vub.ac.be

Diederik Aerts

diraerts@vub.ac.be

Jonito Aerts Arguëlles

jonitoarguelles@gmail.com

Lester Beltran

lestercc21@gmail.com

Suzette Geriente

sgeriente83@yahoo.com

Sandro Sozzo

ss831@le.ac.uk

Tomas Veloz

tveloz@gmail.com

1 Center Leo Apostel for Interdisciplinary Studies, Brussels Free University, Krijgskundestraat 33, 1160 Brussels, Belgium

2 KASK and Conservatory, Jozef Kluyskensstraat 2, 9000 Ghent, Belgium

3 825-C Tayuman Street, Tondo, Manila, The Philippines

4 Block 28 Lot 29 Phase III F1, Kaunlaran Village, Caloocan City, The Philippines

5 School of Business and Research Centre IQSCS, University Road, Leicester LE1 7RH, UK

6 Instituto de Filosofía y Ciencias de la Complejidad IFICC, Los Alerces 3024, Nuñoa, Santiago, Chile 
physics laboratories. This is the first part of a two-part article. In the second part (Aerts et al. in Found Sci, 2017) we present a symmetrized version of the same experiment for which we provide a quantum modeling of the collected data in Hilbert space.

Keywords Human cognition - Quantum structures - Bell's inequalities · Entanglement

\section{Introduction}

Entanglement is one of the most characteristic manifestations of quantum structures and has been widely investigated both theoretically and experimentally. It is nowadays generally recognized that quantum entangled entities may exhibit non-local correlations that cannot be accounted for in a classical probabilistic framework, as formalized by Kolmogorov (1933). Entanglement, however, is not a prerogative of micro-systems only. Its presence can be evidenced also in connected macro-systems (Aerts 1991; Aerts et al. 2000; Sassoli de Bianchi 2013a, b) and in cognitive domains, when certain experiments are performed with human participants (Aerts and Sozzo 2011, 2014).

The scope of the present article is to present a cognitive psychology experiment where participants were interrogated about their preferences on wind directions, and show that there is a remarkable resemblance with typical coincidence experiments on spin entities in entangled states. More precisely, we will show that, when respondents jointly select two wind directions, probabilistically speaking they do so very much like how spin values along those same directions are selected by Stern-Gerlach apparatuses operating on spin bipartite entities in singlet states. This occurs because Bell's inequality (more precisely, its $\mathrm{CHSH}$ version) is violated in both domains with equivalent numerical magnitude, thus allowing to conclude about the detection of entanglement in cognitive experiments, similarly to how it is typically detected in physics experiments.

The article is organized as follows. In Sect. 2, we describe the situation of a typical (EPR-Bohm like) 'Bell-test experiment' in physics, indicating what the quantum mechanical prediction is, as far as the violation of Bell's inequality is concerned. Then, in Sect. 3, we describe our experiment with the conceptual Two different wind directions entity, and its results, highlighting how Bell's inequality is violated in a way that is very similar to as it is violated in physics. Finally, in Sect. 4, we provide some final remarks.

\section{Entanglement in a Typical CHSH (Two-Channel) Experiment}

The seminal studies of John Bell on the foundations of quantum theory showed that a measurement on a quantum entity does not generally reveal a pre-existing value of the measured quantity, which is instead actualized by the measurement context ('quantum contextuality'), and that such process of 'actualization of potential properties' also occurs when the measurement context is formed by parts that are separated by large spatial distances. In other words, quantum contextuality also holds at a distance ('quantum nonlocality'). The latter effect is determined by what physicists call, more specifically, 'quantum entanglement'. Effects due to the presence of quantum entanglement were firstly identified in the seventies of the foregoing century by experiments at the time not yet completely convincing (Freedman and Clauser 1972; Holt 1973; Fry and Thompson 1976; 
Lamehi-Rachti and Mittig 1976), which culminated in 1982 in the major photon correlation experiment performed by the team of Alain Aspect in Paris (Aspect et al. 1982; Aspect 1983). More recent experiments (see, e.g., Aspect et al. 1982; Tittel et al. 1998; Weihs et al. 1998; Genovese 2005; Giustina et al. 2013; Christensen et al. 2013; Hensen et al. 2016) have eventually 'closed all loops', confirming that quantum theory properly describes the situation, thus certifying the 'reality of quantum entanglement as a phenomenon'.

Let us describe the experimental setting of a typical Bell-test experiment, and how it is modeled by quantum theory. A source prepares a bipartite compound entity in a state characterized by an overall spin value equal to zero. Also, the state of the bipartite entity is such that the two sub-entities forming it, which are assumed to be of spin- $\frac{1}{2}$, fly apart in opposite spatial directions. Measuring apparatuses are in regions of space that are located symmetrically with respect to the source, along the direction of propagation of the two subentities, allowing for coincident measurements of their spin values, along given axes. If the spin of the sub-entity moving to the left is measured along the $A$-axis, there will be two possible outcomes: a 'spin up' outcome and a 'spin down' outcome, which will be denoted $A_{1}$ and $A_{2}$, respectively. Similarly, if the spin of the sub-entity moving to the right is measured along the $B$-axis, the 'spin up' and a 'spin down' outcomes will be denoted $B_{1}$ and $B_{2}$, respectively (a schematic representation of the experiment is presented in Fig. 1).

The experiments that have been performed show that the outcomes of the joint spin measurements on the two sub-entities correlate in a very special way, in close accordance with quantum mechanical predictions, thus making a convincing case for the hypothesis that quantum theory provides a faithful modeling of the situation, i.e., that the bipartite entity is in an entangled pre-measurement state. More precisely, the values of the probabilities characterizing these correlations, predicted by quantum theory, are as follows (as reported by manuals of quantum mechanics). If $\alpha$ is the angle between the $A_{1}$ and $B_{1}$ (respectively, $A_{2}$ and $B_{2}, A_{1}$ and $B_{2}, A_{2}$ and $B_{1}$ ) directions, then the probability of finding the $A_{1}$ and $B_{1}$ (respectively, $A_{2}$ and $B_{2}, A_{1}$ and $B_{2}, A_{2}$ and $B_{1}$ ) outcomes is: $\frac{1}{2} \sin ^{2} \frac{\alpha}{2}$.

In concrete experiments, an angle of $45^{\circ}$ is usually chosen between the $A_{1}$ and $B_{1}$ directions and the $A_{2}$ and $B_{2}$ directions, hence there is also an angle of $180^{\circ}-45^{\circ}=135^{\circ}$ between the $A_{1}$ and $B_{2}$ directions and the $A_{2}$ and $B_{1}$ directions, as described in Fig. 1 (the reason for this particular choice is that it produces correlations for which the presence of entanglement becomes most visible). This means that the probability for the $A_{1}$ and $B_{1}$ directions to correlate is $p\left(A_{1}, B_{1}\right)=\frac{1}{2} \sin ^{2} \frac{45^{\circ}}{2}=\frac{1}{8}(2-\sqrt{2}) \approx 0.0732$, and same for $p\left(A_{2}, B_{2}\right)$. Also, the probability for the $A_{1}$ and $B_{2}$ directions to correlate is $p\left(A_{1}, B_{2}\right)=\frac{1}{2} \sin ^{2} \frac{135^{\circ}}{2}=\frac{1}{8}(2+\sqrt{2}) \approx 0.4268$, and same for $p\left(A_{2}, B_{1}\right)$. Note that $p\left(A_{1}, B_{1}\right)+p\left(A_{2}, B_{2}\right)+p\left(A_{1}, B_{2}\right)+p\left(A_{2}, B_{1}\right)=\frac{2}{8}(2-\sqrt{2})+\frac{2}{8}(2+\sqrt{2})=1$, which

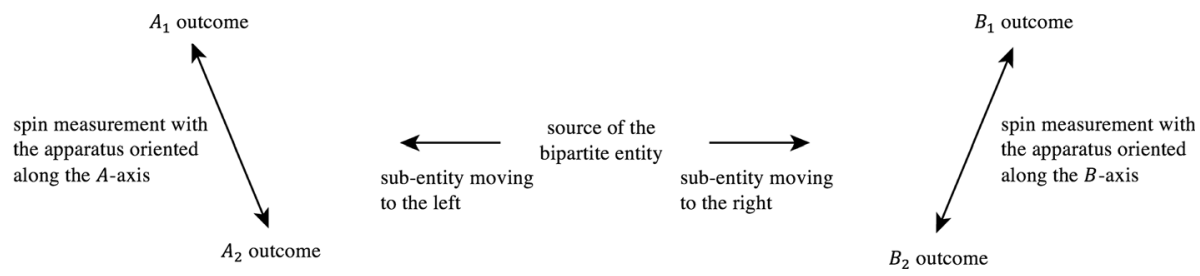

Fig. 1 A schematic representation of a typical Bell test experimental setting in physics 
means that the measurements give rise with certainty to one of the four possible correlations (this is the idealized situation of detectors having $100 \%$ efficiency).

To explain how entanglement can be deduced from the observed correlations, one needs to introduce Bell's inequality (Bell 1964), and more specifically its variant called CHSH inequality, due to Clauser et al. (1969). But first, let us explain which aspect of our physical reality is tested by the latter. If we consider the experimental situation presented in Fig. 1, there is an obvious analogy with ordinary reality that comes to mind, namely the situation of the explosion of a material object into two fragments, one flying to the left and the other to the right. Obviously, these two fragments of the initial material object will manifest different types of correlations. To name an obvious one, if the object has a color, the two fragments will have the same color. The weights of the two fragments will also be perfectly correlated, as their sum must be equal to the weight of the unexploded object. The same is true for their momenta, which for instance will have to sum to zero in case before the explosion the object was at rest. The distances of the fragments from where the explosion took place, at a given moment, are also correlated, in ways that depend on their masses and momenta. Equally so, if rotation is involved, there will be a correlation of the angular momenta, which will be opposite in direction in case the material object had no initial rotational movement.

If we assume that some indeterminism is involved, i.e., that we lack knowledge about the exact initial state of the material object, then the previously mentioned physical quantities associated with the two flying apart fragments are only describable in probabilistic terms, which means that also correlations will be described probabilistically. However, not all combinations of probabilities describing the correlations can make their appearance in situations like the one of the exploding object, and it is precisely this fact, that not all combinations are possible, which is the main content of Bell and CHSH inequalities.

Bell chose to consider expectation values instead of correlation probabilities, which is what we will also do now. When jointly performing measurement $A$ on the left entity and measurement $B$ on the right entity (the situation considered in Fig. 1), we will attribute the value +1 if the two spin outcomes are both 'up' or both 'down' (outcomes $A_{1}$ and $B_{1}$, or $A_{2}$ and $B_{2}$ ), and the value -1 if one of them is 'up' and the other one 'down' (outcomes $A_{1}$ and $B_{2}$, or $A_{2}$ and $B_{1}$ ). The expectation value for the joint measurement is then given by:

$$
E(A, B)=p\left(A_{1}, B_{1}\right)-p\left(A_{1}, B_{2}\right)-p\left(A_{2}, B_{1}\right)+p\left(A_{2}, B_{2}\right) .
$$

Hence, if the angle between $A_{1}$ and $B_{1}$ is $45^{\circ}$, the quantum model predicts the expectation value:

$$
E(A, B)=\frac{2}{8}(2+\sqrt{2})-\frac{2}{8}(2-\sqrt{2})=-\frac{1}{\sqrt{2}} \approx-0.7071 .
$$

To formulate the CHSH inequality, we need to consider, in addition to the joint measurement of $A$ and $B$ illustrated in Fig. 1 (which we will simply denote $A B$ ), three other joint measurements, $A B^{\prime}, A^{\prime} B$ and $A^{\prime} B^{\prime}$, where $A^{\prime}$ is another measurement (different from $A$ ) that can be performed on the left entity and $B^{\prime}$ is another measurement (different from $B$ ) that can be performed on the right entity. Expectation values $E\left(A, B^{\prime}\right), E\left(A^{\prime}, B\right)$ and $E\left(A^{\prime}, B^{\prime}\right)$ can then also be associated to these other three joint measurements, and the $\mathrm{CHSH}$ inequality is: 


$$
|S| \leq 2, \quad S \equiv E(A, B)-E\left(A, B^{\prime}\right)+E\left(A^{\prime}, B\right)+E\left(A^{\prime}, B^{\prime}\right) .
$$

Without entering into a specific discussion of the notion of 'local realism', assumed by Bell to state and prove the 'no-go theorem' associated with the above and similar inequalities, it is sufficient for us to observe that Bell's characterization is such that no bipartite system of the 'material object exploded into two fragments' kind can ever violate (3). To give an example, consider the situation where $A(B)$ corresponds to the measurement of the weight of the left (right) fragment, with the outcome $A_{1}\left(B_{1}\right)$ corresponding to the situation where the weight is more than half the weight of the initial object, and $A_{2}\left(B_{2}\right)$ to the situation where it is less. Also, assuming that the initial object is red, $A^{\prime}\left(B^{\prime}\right)$ is taken to be the measurement of the color of the left (right) fragment, with the outcome $A_{1}\left(B_{1}\right)$ corresponding to the situation where the red color is obtained, and $A_{2}\left(B_{2}\right)$ to the situation where a color different than red is obtained. Clearly, we have the probabilities $p\left(A_{1}^{\prime}, B_{1}^{\prime}\right)=1$ and $p\left(A_{1}, B_{2}^{\prime}\right)=p\left(A_{2}^{\prime}, B_{1}\right)=p\left(A_{2}^{\prime}, B_{2}^{\prime}\right)=0$, since both fragments are necessarily red. Also, since the two fragments cannot both weigh more or less than half the weight of the initial object, we have $p\left(A_{1}, B_{1}\right)=p\left(A_{2}, B_{2}\right)=0$. If $p$ denotes the probability that the initial state of the object is such that the explosion will cause the right fragment to be heavier than the left one, we have: $p\left(A_{2}, B_{1}\right)=p$ and $p\left(A_{1}, B_{2}\right)=1-p$. Also, $p\left(A_{1}^{\prime}, B_{1}\right)=p$ and $p\left(A_{1}, B_{1}^{\prime}\right)=1-p$, and similarly $p\left(A_{1}^{\prime}, B_{2}\right)=1-p$ and $p\left(A_{2}, B_{1}^{\prime}\right)=p$. Replacing these probabilities in the expectation value formulae, we obtain $E(A, B)=-1$, $E\left(A, B^{\prime}\right)=1-2 p, \quad E\left(A^{\prime}, B\right)=-1+2 p \quad$ and $\quad E\left(A^{\prime}, B^{\prime}\right)=1, \quad$ so $\quad$ that $\quad S=-1-$ $1+2 p-1+2 p+1=-2(1-2 p)$. Thus, $|S| \leq 2|1-2 p| \leq 2$, in accordance with (3).

To show that the CHSH inequality is instead violated in spin coincidence measurements, we have to also define the two additional measurements $A^{\prime}$ and $B^{\prime}$ in that context. The latter is chosen to be a spin measurement with the apparatus oriented in such a way that there is an angle of $45^{\circ}$ between directions $A_{1}$ and $B_{2}^{\prime}$, and directions $A_{2}$ and $B_{1}^{\prime}$, hence also an angle of $135^{\circ}$ between directions $A_{1}$ and $B_{1}^{\prime}$, and directions $A_{2}$ and $B_{2}^{\prime}$. On the other hand, $A^{\prime}$ is taken to be such that there is angle of $45^{\circ}$ between directions $A_{1}^{\prime}$ and $B_{1}^{\prime}$, and directions $A_{2}^{\prime}$ and $B_{2}^{\prime}$, hence an angle of $135^{\circ}$ between directions $A_{1}^{\prime}$ and $B_{2}^{\prime}$, and directions $A_{2}^{\prime}$ and $B_{1}^{\prime}$. This means that quantum theory predicts the probabilities $p\left(A_{1}, B_{2}^{\prime}\right)=$ $p\left(A_{2}, B_{1}^{\prime}\right)=p\left(A_{1}^{\prime}, B_{1}\right)=p\left(A_{2}^{\prime}, B_{2}\right)=p\left(A_{1}^{\prime}, B_{1}^{\prime}\right)=p\left(A_{2}^{\prime}, B_{2}^{\prime}\right)=\frac{1}{8}(2-\sqrt{2}) \approx 0.0732$ and $p\left(A_{1}, B_{1}^{\prime}\right)=p\left(A_{2}, B_{2}^{\prime}\right)=p\left(A_{1}^{\prime}, B_{2}\right)=p\left(A_{2}^{\prime}, B_{1}\right)=p\left(A_{1}^{\prime}, B_{2}^{\prime}\right)=p\left(A_{2}^{\prime}, B_{1}^{\prime}\right)=\frac{1}{8}(2+\sqrt{2}) \approx$ 0.4268 . If we attribute again the value +1 for the 'both spin up/down outcomes' and -1 to the 'one spin up and one spin down outcomes', the expectation value of the four joint measurement $A B, A B^{\prime}, A^{\prime} B$ and $A^{\prime} B^{\prime}$ are:

$$
\begin{aligned}
E(A, B) & =p\left(A_{1}, B_{1}\right)-p\left(A_{1}, B_{2}\right)-p\left(A_{2}, B_{1}\right)+p\left(A_{2}, B_{2}^{\prime}\right)=-1 / \sqrt{2} \approx-0.7071 \\
E\left(A, B^{\prime}\right) & =p\left(A_{1}, B_{1}^{\prime}\right)-p\left(A_{1}, B_{2}^{\prime}\right)-p\left(A_{2}, B_{1}^{\prime}\right)+p\left(A_{2}, B_{2}^{\prime}\right)=1 / \sqrt{2} \approx 0.7071 \\
E\left(A^{\prime}, B\right) & =p\left(A_{1}^{\prime}, B_{1}\right)-p\left(A_{1}^{\prime}, B_{2}\right)-p\left(A_{2}^{\prime}, B_{1}\right)+p\left(A_{2}^{\prime}, B_{2}\right)=-1 / \sqrt{2} \approx-0.7071 \\
E\left(A^{\prime}, B^{\prime}\right) & =p\left(A_{1}^{\prime}, B_{1}^{\prime}\right)-p\left(A_{1}^{\prime}, B_{2}^{\prime}\right)-p\left(A_{2}^{\prime}, B_{1}^{\prime}\right)+p\left(A_{2}^{\prime}, B_{2}^{\prime}\right)=-1 / \sqrt{2} \approx-0.7071,
\end{aligned}
$$

so that we obtain the following violation of (3):

$$
|S|=\left|E(A, B)-E\left(A, B^{\prime}\right)+E\left(A^{\prime}, B\right)+E\left(A^{\prime}, B^{\prime}\right)\right|=2 \sqrt{2} \approx 2.8284 .
$$

In other words, the quantum mechanical prediction, nowadays confirmed by a considerable amount of experimental data (Aspect et al. 1982; Tittel et al. 1998; Weihs et al. 1998; 
Genovese 2005; Giustina et al. 2013; Christensen et al. 2013; Hensen et al. 2016), is that the CHSH inequality is violated when joint measurements are performed on bipartite systems, like spin systems, prepared in an entangled state. This is a situation that cannot be properly modeled by a classical (Kolmogorovian) probability theory. However, this doesn't mean that the CHSH inequality cannot be violated also by classical macroscopic systems. For this, it is sufficient that the two components remain connected in some way, so that the left and right measurements can influence each other's states and therefore outcomes (Aerts et al. 2017, 2000; Sassoli de Bianchi 2013a, b; Aerts and Sassoli de Bianchi 2016). This is what can be expected to happen also with entangled quantum entities, although their connection remains in this case hidden, i.e., appears to be a "nonspatial connection', hence the strangeness of the quantum entanglement phenomenon, famously referred to by Einstein as "spooky action at a distance".

\section{Entangled Wind Directions in Human Cognition}

In this section, we consider joint measurements performed on a conceptual entity formed by the combination of two concepts, to highlight the presence of entanglement in human cognition and its similarity with entanglement of micro-physical bipartite entities. More precisely, we consider the following combination of concepts: Two different wind directions. If analyzed from the perspective of its meaning, it is a combination of the following two conceptual elements: One wind direction and Another wind direction. However, in the English language, this combination is usually expressed by the sentence: Two different wind directions. Our investigation of this conceptual combination is to consider measurements that can be performed on the two composing concepts, and analyze the statistics of outcomes associated with the combinations of these measurements. Indeed, it is in this statistics that traces can be be found of the presence of a quantum structure of the entanglement kind.

To explain how measurements are introduced and analyzed, let us first consider the single conceptual element One wind direction, which together with the element Another wind direction is part of the combination Two different wind directions. A typical measurement is to ask a human participant to choose one among two possible wind directions. For example, either the directions North or the direction South, giving rise to a twooutcome measurement that we will denote $A$. To perform a typical Bell-test experiment, we need to define three additional measurements: $A^{\prime}, B$ and $B^{\prime}$. Measurement $A^{\prime}$ is also considered to apply to the conceptual entity One wind direction, and consists in choosing among the two wind directions East and West. Measurement $B$ and $B^{\prime}$ are instead considered to apply to the conceptual entity Another wind direction, and consists in asking a human participant to choose between the directions Northeast and Southwest, and Southeast and Northwest, respectively (see Fig. 2).

To be in the situation where the CHSH inequality can be tested, we need then to consider the joint measurements that can be defined by combining the above four measurements. More specifically, we denote $A B$ the joint measurement of $A$ combined with $B$, $A B^{\prime}$ the joint measurement of $A$ combined with $B^{\prime}, A^{\prime} B$ the joint measurement of $A^{\prime}$ combined with $B$, and $A^{\prime} B^{\prime}$ the joint measurement of $A^{\prime}$ combined with $B^{\prime}$. These are measurements that are now to be performed on the combined concept Two different wind directions. More precisely, joint measurement $A B$ consists of a human subject choosing one among four possible outcomes that are combinations of the outcomes of measurements 


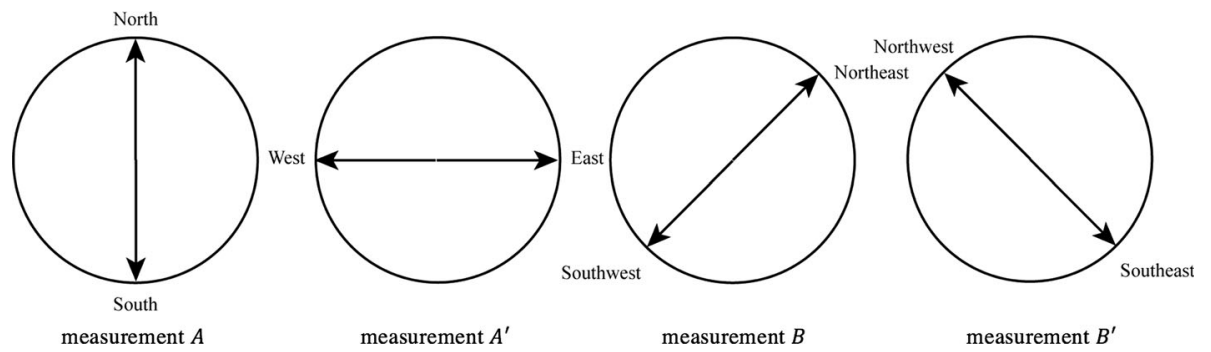

Fig. 2 A graphical representation of the two outcomes for the four measurements $A, A^{\prime}, B$ and $B^{\prime}$

$A$ and $B$. Hence, the possible outcomes of $A B$ are North and Northeast [outcome $\left(A_{1}, B_{1}\right)$ ], North and Southwest [outcome $\left.\left(A_{1}, B_{2}\right)\right]$, South and Northeast [outcome $\left.\left(A_{2}, B_{1}\right)\right]$, and South and Southwest [outcome $\left(A_{2}, B_{2}\right)$ ]. Similarly, joint measurement $A B^{\prime}$ consists of a human subject choosing one among four possible outcomes that are combinations of the outcomes of measurements $A$ and $B^{\prime}$. Hence, the possible outcomes of $A B^{\prime}$ are North and Southeast [outcome $\left(A_{1}, B_{1}^{\prime}\right)$ ], North and Northwest [outcome $\left(A_{1}, B_{2}^{\prime}\right)$ ], South and Southeast [outcome $\left(A_{2}, B_{1}^{\prime}\right)$ ], and South and Northwest [outcome $\left(A_{2}, B_{2}^{\prime}\right)$ ]. Also, joint measurement $A^{\prime} B$ consists of a human subject choosing one among four possible outcomes that are combinations of the outcomes of measurements $A^{\prime}$ and $B$. Hence, the possible outcomes of $A^{\prime} B$ are East and Northeast [outcome $\left(A_{1}^{\prime}, B_{1}\right)$ ], East and Southwest [outcome $\left.\left(A_{1}^{\prime}, B_{2}\right)\right]$, West and Northeast [outcome $\left(A_{2}^{\prime}, B_{1}\right)$ ], and West and Southwest [outcome $\left.\left(A_{2}^{\prime}, B_{2}\right)\right]$. Finally, joint measurement $A^{\prime} B^{\prime}$ consists of a human subject choosing one among four possible outcomes that are combinations of the outcomes of measurements $A^{\prime}$ and measurement $B^{\prime}$. Hence, the possible outcomes of $A^{\prime} B^{\prime}$ are East and Southeast [outcome $\left(A_{1}^{\prime}, B_{1}^{\prime}\right)$ ], East and Northwest [outcome $\left(A_{1}^{\prime}, B_{2}^{\prime}\right)$ ], West and Southeast [outcome $\left(A_{2}^{\prime}, B_{1}^{\prime}\right)$ ], and West and Northwest [outcome $\left(A_{2}^{\prime}, B_{2}^{\prime}\right)$ ]; see Fig. 3.

Having described the four joint measurements $A B, A B^{\prime}, A^{\prime} B$ and $A^{\prime} B^{\prime}$, let us now explain how they were performed in practice. Concerning 'participants and design', we asked 85 persons, chosen at random among colleagues and friends, to fill in a questionnaire with closed-ended questions. The experimental design was a 'repeated measures', or 'within subjects' design, which means that all participants were subject to the same questions and experimental conditions. Concerning 'procedure and materials', the questionnaire consisted in four sequential tests, where each test is a question with four possible answers, with the possibility to only pick one answer. The different answers were accompanied by their graphical description. For instance, the answer "North and Northeast" was associated with the first drawing of Fig. 3, the answer "North and Southwest" with the second drawing of Fig. 3, and so on. More precisely, the first test (denoted test A) corresponded to the joint measurement $A B$, the second test (denoted test $\mathrm{B}$ ) to the joint measurement $A B^{\prime}$, the third test (denoted test $\mathrm{C}$ ) to the joint measurement $A^{\prime} B$, and the last test (denoted test $\mathrm{D}$ ), to the joint measurement $A^{\prime} B^{\prime}$. Before executing these four tests, an introductory text was also presented to the participants, which is the following:

This study has to do with what we have in mind when we use words that refer to categories, and more specifically 'how we think about examples of categories'. Let us illustrate what we mean. Consider the category 'fruit'. Then 'orange' and 'strawberry' are two examples of this category, but also 'fig' or 'olive' are examples 


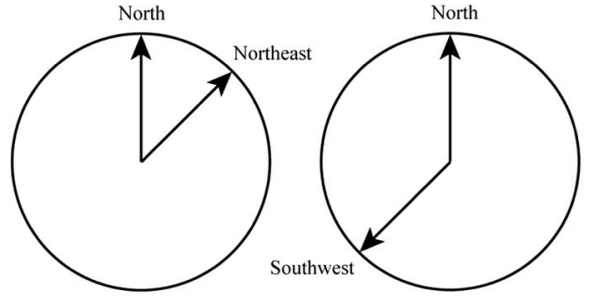

outcome $\left(A_{1}, B_{1}\right)$ outcome $\left(A_{1}, B_{2}\right)$

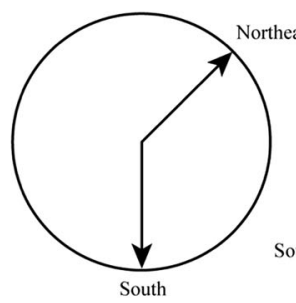

outcome $\left(A_{2}, B_{1}\right)$

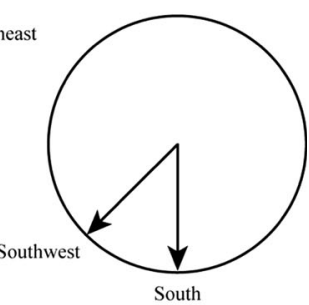

outcome $\left(A_{2}, B_{2}\right)$
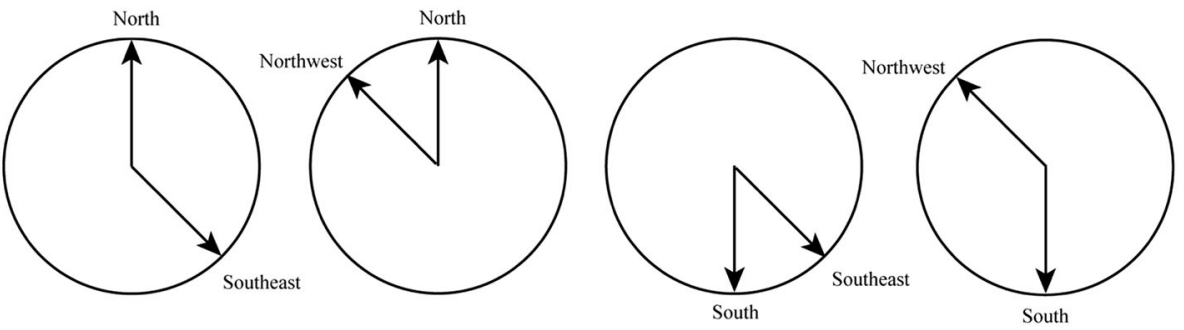

outcome $\left(A_{1}, B^{\prime}{ }_{1}\right)$

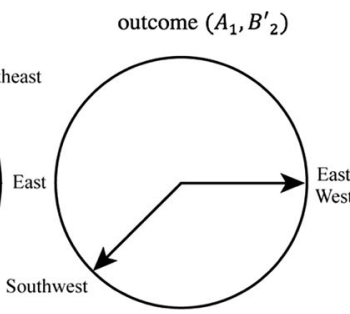

outcome $\left(A_{2}, B^{\prime}{ }_{1}\right)$

outcome $\left(A_{2}, B^{\prime}{ }_{2}\right)$

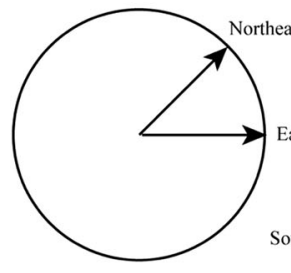

outcome $\left(A^{\prime}, B_{1}\right)$

outcome $\left(A_{1}^{\prime}, B_{2}\right)$

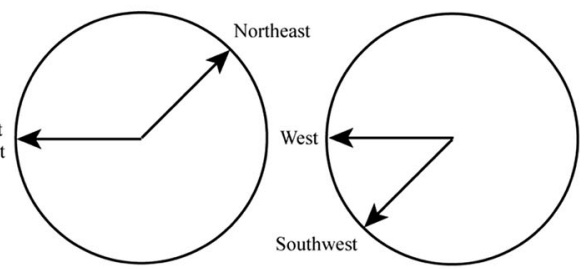

outcome $\left(A^{\prime}{ }_{2}, B_{1}\right)$

outcome $\left(A^{\prime}{ }_{2}, B_{2}\right)$

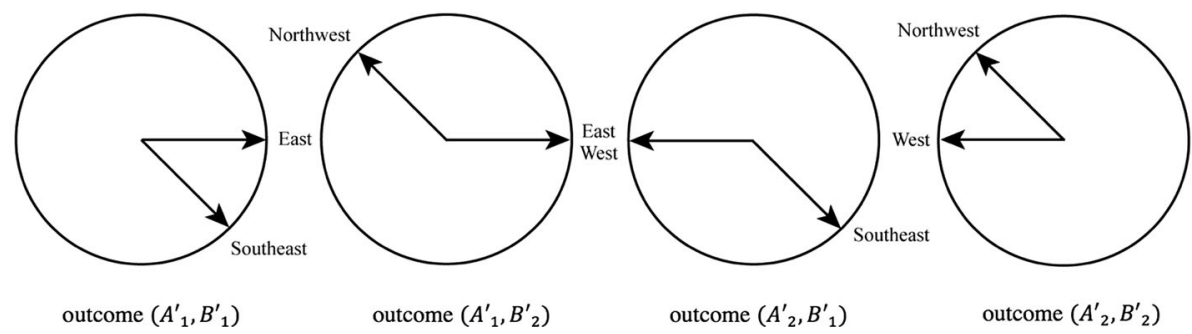

Fig. 3 A graphical representation of the four possible outcomes of the joint measurements $A B, A B^{\prime}, A^{\prime} B$ and $A^{\prime} B^{\prime}$

of the same category. In each test of the questionnaire you will be asked to pick one of the examples of a set of given examples for a specific category. And we would like you to pick that example that you find 'the best example' of the category. In case there are more than one example which you find the best example, pick then the one you prefer anyhow in some way. In case there are two examples which you both find the best, and hence hesitate which ones to take, just take then the one you slightly prefer, however slight the preference might be. In case you really have no preference, you are allowed to pick at random, and even use a coin to do so. It is mandatory that you always 'pick one and only one example'. So, one of the tests could be that the 
category 'fruit' is given, and you are asked to pick one of the examples 'orange', 'strawberry', 'fig' or 'olive', and hence choose amongst these the one you find 'the best example'. What is also very important, let all aspects of yourself play a role in the choice you make, ratio, but also imagination, feeling, emotion, and whatever. Hence, suppose you feel like choosing a specific example as the best, but your ratio argues, eventually after analysis, that it is another one which is objectively the best, and then you are allowed (not obliged however) to stay with your feeling, and not follow the analysis. There are four tests, test A, test B, test $\mathrm{C}$, and test $\mathrm{D}$, explained on the next four pages, and hence for each of them one choice to be made.

In addition to that, each single test was preceded by the following text: "Consider the sentence Two different wind directions and choose which of the following examples is the best example of Two different wind directions. In case you find more than one best example, pick the one you find really the best, whatever aspect of yourself tells you that this is the one you prefer. You must pick one and only one, even in case you cannot decide after a while. If this would happen, namely that you keep hesitating, choose then one at random (you may use a coin for this). Put a cross behind the one you choose."

For each one of the four tests, that is, for each one of the four joint measurements $A B$, $A B^{\prime}, A^{\prime} B$ and $A^{\prime} B^{\prime}$, we then calculated from the collected data the relative frequencies of the different outcomes (which in the large number limit can be interpreted as probabilities). The obtained experimental values are:

$$
\begin{array}{llll}
p\left(A_{1}, B_{1}\right)=0.13, & p\left(A_{1}, B_{2}\right)=0.55, & p\left(A_{2}, B_{1}\right)=0.25, & p\left(A_{2}, B_{2}\right)=0.07 \\
p\left(A_{1}, B_{1}^{\prime}\right)=0.47, & p\left(A_{1}, B_{2}^{\prime}\right)=0.12, & p\left(A_{2}, B_{1}^{\prime}\right)=0.06, & p\left(A_{2}, B_{2}^{\prime}\right)=0.35 \\
p\left(A_{1}^{\prime}, B_{1}\right)=0.13, & p\left(A_{1}^{\prime}, B_{2}\right)=0.38, & p\left(A_{2}^{\prime}, B_{1}\right)=0.42, & p\left(A_{2}^{\prime}, B_{2}\right)=0.07 \\
p\left(A_{1}^{\prime}, B_{1}^{\prime}\right)=0.09, & p\left(A_{1}^{\prime}, B_{2}^{\prime}\right)=0.44, & p\left(A_{2}^{\prime}, B_{1}^{\prime}\right)=0.38, & p\left(A_{2}^{\prime}, B_{2}^{\prime}\right)=0.09,
\end{array}
$$

so that the corresponding expectation values are:

$$
\begin{gathered}
E(A, B)=p\left(A_{1}, B_{1}\right)-p\left(A_{1}, B_{2}\right)-p\left(A_{2}, B_{1}\right)+p\left(A_{2}, B_{2}\right)=-0.6 \\
E\left(A, B^{\prime}\right)=p\left(A_{1}, B_{1}^{\prime}\right)-p\left(A_{1}, B_{2}^{\prime}\right)-p\left(A_{2}, B_{1}^{\prime}\right)+p\left(A_{2}, B_{2}^{\prime}\right)=0.65 \\
E\left(A^{\prime}, B\right)=p\left(A_{1}^{\prime}, B_{1}\right)-p\left(A_{1}^{\prime}, B_{2}\right)-p\left(A_{2}^{\prime}, B_{1}\right)+p\left(A_{2}^{\prime}, B_{2}\right)=-0.6 \\
E\left(A^{\prime}, B^{\prime}\right)=p\left(A_{1}^{\prime}, B_{1}^{\prime}\right)-p\left(A_{1}^{\prime}, B_{2}^{\prime}\right)-p\left(A_{2}^{\prime}, B_{1}^{\prime}\right)+p\left(A_{2}^{\prime}, B_{2}^{\prime}\right)=-0.62 .
\end{gathered}
$$

It follows that we find the following violation of the CHSH inequality:

$$
|S|=\left|E(A, B)-E\left(A, B^{\prime}\right)+E\left(A^{\prime}, B\right)+E\left(A^{\prime}, B^{\prime}\right)\right|=2.47 .
$$

Equation (7) shows a striking similarity with the values of the violation obtained in typical physics experiments designed to detect entanglement and non-locality in spin-like coincidence measurements on pairs of quantum entities (electrons, ions, photons). For example, according to Genovese (2005), Aspect et al. found $|S|=2.697 \pm 0.015$, Tittel et al. found $|S|=2.38 \pm 0.16$, Weihs et al. found $|S|=2.73 \pm 0.02$, Aspelmeyer et al. found $|S|=2.41 \pm 0.10$, Pittman and Franson found $|S|=2.44 \pm 0.13$, and Peng et al. found $|S|=2.45 \pm 0.09$. Also, Hensen et al. (2016) found $|S|=2.42 \pm 0.20$.

Note that we performed a statistical analysis of our experimental data, to test whether the observed deviation from the value $|S|=2$ was only due to chance. To this end, we computed a one tail one sample $t$ test for means of the experimental values of $|S|$ in Equation (7), against the constant value 2, finding a $p$-value $p(d f=84)=0.05$. This is a 
borderline result with respect to the rejection of the null hypothesis that the two means are equal in the $t$ test, but most probably this is only due to the reduced size of the statistical sample.

\section{Concluding Remarks}

The results presented in the previous section show that conceptual entities can violate Bell's inequality (here the CHSH version of it) and that the magnitude of the violation is the same as that obtained in physics experiments with entangled pairs of spin- $\frac{1}{2}$ entities (or similar quantum entities, like entangled photons). This closeness of values between cognitive and physics experiments is particularly striking because 'joint spin measurements' and 'joint wind measurements' are both about actualizing potential spatial directions. This means that people appear to actualize spatial directions (here in association with winds) in a way that is remarkably similar, statistically speaking, to how spin directions (more precisely, 'up' and 'down' directions, along given axes) are actualized by physical apparatuses, like Stern-Gerlach apparatuses.

We mentioned in Sect. 2 that quantum entanglement can be understood as being the result of a connection between the two sub-entities forming the bipartite system. It is worth mentioning that this interpretation is supported by the 'extended Bloch representation of quantum mechanics', where entangled states of bipartite systems can be written in a way that the two sub-systems always remain in well-defined states, their entanglement being instead associated with a third 'element of reality', corresponding to the emergence of a non-spatial connection between them (Aerts and Sassoli de Bianchi 2016) (which cannot be deduced from the states of the sub-entities, in accordance with the principle that the whole is greater than the sum of its parts). Coincidence measurements can therefore be understood as processes during which the symmetry of such connection between the subentities is broken, bringing them in a condition of (temporary) separation. This 'symmetry breaking' (the actual breaking the symmetry of the potential) is a process that genuinely creates correlations that were not present before the measurement (called 'correlations of the second kind' Aerts 1991), and it is precisely such process of 'creation of correlations', as opposed to a process of mere 'discovery of already existing correlations' (called 'correlations of the first kind', as in the situation of the exploded object described in Sect. 2) that is responsible for the violation of the CHSH inequality.

When dealing with entanglement in human cognition, the nature of the non-spatial connection responsible for the violation of the $\mathrm{CHSH}$ inequality can be understood as a 'connection through meaning'. Indeed, before asking the participants to choose a pair of specific directions, an abstract meaning-connection undoubtedly exists between the two conceptual wind directions, expressing all their possible concrete actualizations (instantiations) that are meaningful for us humans, in view of our experience with Euclidean space and its directions. And when pairs of specific directions are actualized, the symmetry of such abstract meaning-connection is broken, creating in this way correlations.

We conclude by evoking an important aspect in the violation of Bell's and CHSH inequalities: the preservation of so-called 'marginal law', which is typically assumed in their derivation (Fine 1982). ${ }^{1}$ Some authors consider that the violation of the inequalities is

\footnotetext{
1 The marginal law, in the ambit of Bell-test experiments, expresses the classical Kolmogorovian requirement that the following eight equalities must be fulfilled: $\sum_{j=1}^{2} p\left(A_{i}, B_{j}\right)=\sum_{j=1}^{2} p\left(A_{i}, B_{j}^{\prime}\right), i=1,2$;
} 
not conclusive of the presence of entanglement, if the marginal law is also violated (Dzhafarov and Kujala 2014; Dzhafarov et al. 2016). Without going into the specifics of this issue, we observe the following. The data we presented in this article do violate the marginal law, although not in a very pronounced way. This does not mean, however, that they cannot be modeled in a quantum theoretical way. For this, it is sufficent to introduce 'entangled measurements' in addition to 'entangled states', as was done in the modeling of previous cognitive experiments, also revealing the presence of entanglement in human cognition (Aerts and Sozzo 2014). But more importantly, it is possible to show that the violation of the marginal law in our experiment is not fundamental, but accidental, therefore in no way indicative of the fact that one cannot conclude about the presence of genuine entanglement effects revealed by our data.

Indeed, measurement $A$ was taken to correspond to the South-North axis, with measurement $B$ then rotated of an angle of $45^{\circ}$ clockwise with respect to $A$, corresponding to the Southwest-Northeast axis, and measurements $A^{\prime}$ and $B^{\prime}$ rotated of an angle of $90^{\circ}$ clockwise with respect to $A$ and $B$, corresponding to the East-West and SoutheastNorthwest axes, respectively. This specific choice of axis for the $A$-measurement (and consequently for the other rotated measurements) introduced a distinction (a first symmetry breaking) between the concept of 'spatial direction' and the more specific concept of 'wind direction'. However, a symmetrized version of the Two different wind directions experiment can be designed, whose data will then obey the marginal law but still violate the CHSH inequality with same magnitude. This will be explained in detail in the second part of our article (Aerts et al. 2017), where an explicit quantum modeling in Hilbert space of the experimental data will also be given, using a singlet state to describe the initial state of the Two different wind directions conceptual entity, and product measurements to describe the four different joint measurements that are executed on it.

Open Access This article is distributed under the terms of the Creative Commons Attribution 4.0 International License (http://creativecommons.org/licenses/by/4.0/), which permits unrestricted use, distribution, and reproduction in any medium, provided you give appropriate credit to the original author(s) and the source, provide a link to the Creative Commons license, and indicate if changes were made.

\section{References}

Aerts, D. (1991). A mechanistic classical laboratory situation violating the Bell inequalities with $2 \sqrt{2}$, exactly 'in the same way' as its violations by the EPR experiments. Helvetica Physica Acta, 64, 1-23.

Aerts, D., Aerts, S., Broekaert, J., \& Gabora, L. (2000). The violation of Bell inequalities in the macroworld. Foundations of Physics, 30, 1387-1414.

Aerts, D., Arguëlles, J. A., Beltran, L., Geriente, S., Sassoli de Bianchi, M. \& Sozzo, S. (2017). Spin and wind directions II: A Bell state quantum model. Foundations of Science. doi:10.1007/s10699-0179530-2.

Aerts, D., \& Sassoli de Bianchi, M. (2016). The extended bloch representation of quantum mechanics. Explaining superposition, interference and entanglement. Journal of Mathematical Physics, 57, 122110.

Aerts, D., \& Sozzo, S. (2011). Quantum structure in cognition. Why and how concepts are entangled. In Quantum interaction 2011. Lecture notes in computer science, 7052 (pp. 116-127). Berlin: Springer.

Footnote 1 continued
$\begin{aligned} & \sum_{i=1}^{2} p\left(A_{i}, B_{j}\right)=\sum_{i=1}^{2} p\left(A_{i}^{\prime}, B_{j}\right), \quad j=1,2 ; \quad \sum_{j=1}^{2} p\left(A_{i}^{\prime}, B_{j}\right)=\sum_{j=1}^{2} p\left(A_{i}^{\prime}, B_{j}^{\prime}\right), \quad i=1,2 ; \\ & \sum_{i=1}^{2} p\left(A_{i}, B_{j}^{\prime}\right)=\sum_{i=1}^{2} p\left(A_{i}^{\prime}, B_{j}^{\prime}\right), j=1,2 .\end{aligned}$


Aerts, D., \& Sozzo, S. (2014). Quantum entanglement in conceptual combinations. International Journal of Theoretical Physics, 53, 3587-3603.

Aspect, A. (1983). Trois tests expérimentaux des inégalités de Bell par mesure de corrélation de polarization de photons. Orsay: Thèse d'Etat.

Aspect, A., Dalibard, J., \& Roger, G. (1982). Experimental test of Bell's inequalities using time-varying analyzers. Physical Review Letters, 49, 1804-1807.

Aspect, A., Grangier, P., \& Roger, G. (1982). Experimental realization of Einstein-Podolsky-Rosen-Bohm Gedankenexperiment: A new violation of Bell's inequalities. Physical Review Letters, 49, 91-94.

Bell, J. S. (1964). On the Einstein-Podolsky-Rosen paradox. Physics, 1, 195-200.

Christensen, B. G., McCusker, K. T., Altepeter, J., Calkins, B., Gerrits, T., Lita, A., et al. (2013). Detectionloophole-free test of quantum nonlocality, and applications. Physical Review Letters, 111, 1304-1306.

Clauser, J. F., Horne, M. A., Shimony, A., \& Holt, R. A. (1969). Proposed experiment to test local hiddenvariable theories. Physical Review Letters, 23, 880-884.

Dzhafarov, E. N., \& Kujala, J. V. (2014). Selective influences, marginal selectivity, and Bell/CHSH inequalities. Topics in Cognitive Science, 6, 121-128.

Dzhafarov, E. N., Kujala, J. V., Cervantes, V. H., Zhang, R., \& Jones, M. (2016). On contextuality in behavioural data. Philosophical Transactions of the Royal Society A: Mathematical, Physical and Engineering Sciences. doi:10.1098/rsta.2015.0234.

Fine, A. (1982). Joint distributions, quantum correlations, and commuting observables. Journal of Mathematical Physics, 23, 1306-1310.

Freedman, S. J., \& Clauser, J. F. (1972). Experimental test of local hidden variable theories. Physical Review Letters, 28, 938-941.

Fry, E. S., \& Thompson, R. C. (1976). Experimental test of local hidden-variable theories. Physical Review Letters, 37, 465-468.

Genovese, M. (2005). Research on hidden variable theories. A review of recent progresses. Physics Reports, $413,319-396$.

Giustina, M., Mech, A., Ramelow, S., Wittmann, B., Kofler, J., Beyer, J., et al. (2013). Bell violation using entangled photons without the fair-sampling assumption. Nature, 497, 227-230.

Hensen, B., Bernien, H., Dréau, A. E., Reiserer, A., Kalb, N., Blok, M. S., et al. (2016). Loophole-free Bell inequality violation using electron spins separated by 1.3 kilometres. Nature, 526, 682-686.

Holt, R. A. (1973). Atomic cascade experiments. Ph.D. thesis, Physics Department, Harvard University.

Kolmogorov, A. N. (1933). Grundbegriffe der Wahrscheinlichkeitrechnung, Ergebnisse Der Mathematik; translated as Foundations of Probability (p. 1950). New York: Chelsea Publishing Company.

Lamehi-Rachti, M., \& Mittig, W. (1976). Quantum mechanics and hidden variables: A test of Bell's inequality by the measurement of spin correlation in low-energy proton-proton scattering. Physical Review D, 14, 2543-2555.

Sassoli de Bianchi, M. (2013a). Quantum dice. Annals of Physics, 336, 56-75.

Sassoli de Bianchi, M. (2013b). Using simple elastic bands to explain quantum mechanics: A conceptual review of two of Aerts' machine-models. Central European Journal of Physics, 11, 147-161.

Tittel, W., Brendel, J., Zbinden, H., \& Gisin, N. (1998). Violation of Bell's inequalities by photons more than $10 \mathrm{~km}$ apart. Physical Review Letters, 81, 3563-3566.

Weihs, G., Jennewein, T., Simon, C., Weinfurter, H., \& Zeilinger, A. (1998). Violation of Bell's inequality under strict Einstein locality condition. Physical Review Letters, 81, 5039-5043.

Diederik Aerts is theoretical physicist and professor at the Brussels Free University where he is director of the Center Leo Apostel for Interdisciplinary Studies. His research interests concern the foundations of quantum physics and the foundations of cognition. He currently works in developing quantum models for non classical probabilistic phenomena in cognition (e.g., guppy effect, conjunction fallacy, disjunction effect) and for decision making under uncertainty, with applications in cognitive science, computer science and economics. Together with some of his collaborators he is considered to be one of the founding researchers of the emergent scientific domain called Quantum Cognition. He is the author of more than 200 publications in international peer reviewed journals and the organizer of several international conferences. As such he was the scientific and artistic coordinator of the Einstein meets Magritte conference, where the world's leading scientists and artists gathered to reflect about science, nature, human action and society.

Jonito Aerts Arguëlles has a Bachelor in Visual Arts and is bound to finish a Master in Visual Arts at the KASK of the School of Arts in Ghent, Belgium. His interest is the interface between art and science and he actually investigates experimental settings related to ephemeral visual phenomena, such as the afterimage. In his collaboration with the Brussels group on quantum cognition he explores the ways in which the non 
classical phenomena can be identified in communications different from language, related to art and science (e.g., vision), but also in interactions of the human mind with the entities of the emerging Web of Things. In that respect, he participated in recent investigations of the Brussels group on the presence of quantum effects in the meaning structures in human cognition and written texts.

Lester Beltran has a Bachelor in Computer Science from Interface Computer College in the Philippines and is currently completing a Master in Mechanical Engineering at the Wrexham Gwyndŵr University in the United Kingdom. His interest is in artistic and mechanical design, graphical design, and the decision processes connected with complex designs, which is how he came into contact with the models of decision making developed in quantum cognition. He is also interested in aspects of natural language processing, hence his collaboration with the Brussels group on quantum cognition, investigating the application of their quantum approach to human cognition in the modeling of large corpora of texts, such as the World Wide Web.

Suzette Geriente has a Bachelor of Science in Psychology from the Philippine Normal University and a Master of Arts in Education from the University of Luzon in the Philippines. Her interest lies in the structure of human cognition and the question of the role played by quantum models has more specifically intrigued her. This is how she came into contact with the Brussels group on quantum cognition and because of her expertise in experimental psychology she participated in the development of experiments testing the presence of quantum structures in human cognition. She is also interested in decision-making under uncertainty in economics and the recent investigations of non classical cognitive science phenomena such as the conjunction fallacy within the meaning structures encountered in written documents.

Massimiliano Sassoli de Bianchi graduated in physics from the University of Lausanne (UNIL), Switzerland, in 1989. From 1990 to 1991, he was an Assistant in the Department of Theoretical Physics (DPT) of the University of Geneva (UNIGE). In 1992, he joined the Institute of Theoretical Physics (IPT) at the Federal Institute of Technology in Lausanne (EPFL), as a Ph.D. candidate. He received the Ph.D. degree in physics from EPFL in 1995. Since 1996, he has been working in the private sector and as an independent researcher. In 2016, he joined the Leo Apostel Centre for Interdisciplinary Studies (CLEA), at the Brussels Free University (Vrije Universiteit Brussel-VUB), as a research fellow. He is also director of the Laboratorio di Autoricerca di Base (LAB), in Switzerland, and Editor of its journal AutoRicerca. His research activities are focused on the foundations of physics, cognitive science, and consciousness studies.

Sandro Sozzo is associate professor at the School of Business of the University of Leicester. His research interests are highly interdisciplinary and mainly concern the development of novel mathematical models of cognition and decision-making under uncertainty, with relevant applications in computer science, economics and finance. He is author of more than 80 publications, organized conferences, managed special issues as guest editor, and was invited to give lectures in UK (Oxford), US, Canada, Europe and China. He is the director of the "Research Centre for Quantum Social and Cognitive Science" (IQSCS), the secretary of the "International Quantum Structures Association" (IQSA) and the managing editor of the Springer Nature journal "Foundations of Science".

Tomas Veloz graduated in physics (B.Sc.), mathematics (B.Sc.), and computer science (M.Sc.) from the University of Chile, in 2005, 2007 and 2010, respectively. He received the Ph.D. degree in interdisciplinary studies from the University of British Columbia in 2015. During his Ph.D., in 2013, he joined the Leo Apostel Centre for Interdisciplinary Studies (CLEA), at the Brussels Free University (Vrije Universiteit Brussel-VUB), as a research fellow, and since 2014 he has been the director of the Systemics department of the Institute of Philosophy and Complexity Sciences (IFICC) in Santiago, Chile. In 2016-2017 he worked as a postdoctoral researcher at CLEA. He is currently a postdoctoral researcher at IFICC. 